\title{
Derivativos sobre Commodities Influenciam a Volatilidade dos Preços à Vista? Uma análise nos mercados de boi gordo e café arábica no Brasil
}

\author{
Rodrigo Lanna Franco da Silveira', Leandro Maciel² e \\ Rosangela Ballini ${ }^{3}$
}

Resumo: Além de fatores conjunturais da economia mundial e estruturais relativos à oferta e demanda global por commodities, aponta-se que o movimento altista dos preços destes produtos na década de 2000 pode também ser explicado pelo maior contágio dos derivativos nos seus respectivos mercados à vista. Argumenta-se ainda que esses papéis contribuíram para a elevação da volatilidade das cotações spot. Neste contexto, este artigo avaliou a influência das negociações e da volatilidade dos preços futuros sobre a volatilidade dos preços à vista nos mercados de café arábica e de boi gordo no Brasil. Realizaram-se testes de causalidade de Granger, decomposição da variância do erro de previsão, considerando modelos VAR, além de testes de causalidade na variância, baseados na função de correlação cruzada e no multiplicador de Lagrange. Os resultados mostraram que, em geral, variações não esperadas do volume de negociação e variabilidade dos preços futuros alteraram o padrão de volatilidade dos respectivos mercados spot.

Palavras-chaves: Mercados futuros, commodities, volatilidade, causalidade.

Abstract: The bullish movement in commodity prices during the 2000s can be explained based on structural and conjectural factors. In addition, it was argued that this price movement was amplified by the contagion from the derivative markets. In this context, these contracts were one of the aspects responsible for an increase in cash price volatility. Thus, this paper evaluated the influence of trading activity (volume and open interest) and futures price volatility in spot price volatility for arabica coffee and live cattle in Brazilian markets. Granger causality tests, forecast error variance decomposition, considering vector autoregression models, and tests of causality in variance, based on the cross-correlation

1. Professor no Instituto de Economia da Universidade Estadual de Campinas - IE/Unicamp. E-mail: rlanna@unicamp.br

2. Doutorando em Engenharia Elétrica na Faculdade de Engenharia Elétrica e Computação da Universidade Estadual de Campinas - FEEC/Unicamp. E-mail: maciel@dca.fee.unicamp.br

3. Professora no Instituto de Economia da Universidade Estadual de Campinas - IE/Unicamp. E-mail: ballini@eco.unicamp.br 
function and on the idea of Lagrange multiplier were conducted. The results showed that, during the period considered, in most cases, an unexpected movement in trading volume and variability of futures prices changed the pattern of spot price volatility.

Key-words: Futures markets, commodities, volatility, causality.

Classificação JEL: Q14, G13, G32.

\section{Introdução}

A introdução e o significativo crescimento das negociações de derivativos sobre ativos financeiros e commodities em diferentes países do mundo têm produzido um controverso debate na literatura econômica e financeira acerca das influências destes papéis sobre as cotações nos mercados à vista.

Os contratos de derivativos, além de permitirem a realização de operações de hedge e diversificação de carteiras de investimento, possuem importante função econômica baseada na descoberta de preços ${ }^{4}$. Por um lado, argumenta-se que o processo de descoberta de preços eleva a eficiência do mercado ao tornar possível um aumento no fluxo de informações, contribuindo para uma queda na volatilidade das cotações à vista (MORGAN, 1999; SANTOS, 2002; SRINIVASAN e BHAT, 2008; DEBASISH, 2009). Os futuros e as opções, por possibilitarem alta alavancagem a custo reduzido, atraem traders bem informados, os quais atuavam apenas nos mercados spot, reduzindo a assimetria nas informações.

4. A partir da oferta e demanda por contratos futuros, os preços futuros são formados, sendo estes então reflexos do consenso dos agentes de mercado sobre a cotação spot do ativo objeto do contrato para uma data futura. Este processo de formação dos preços é caracterizado por grande dinamismo, dado que as informações são incorporadas e processadas com grande velocidade. Dessa forma, os agentes de mercado utilizam os preços futuros para a formação das cotações à vista, o que acaba por permitir o planejamento estratégico da atividade.
Por outro lado, outros trabalhos apontam para uma direção contrária, ou seja, o surgimento e a evolução dos mercados de derivativos têm sido determinantes para a desestabilização das cotações no mercado spot (FIGLEWSKI, 1981; STEIN, 1987). A principal razão de tal fato está associada à alta alavancagem que estes contratos permitem obter a um custo relativamente pequeno se comparado às transações à vista, o que acaba atraindo diversos tipos de especuladores, sendo parte deles dotada de poucas informações a respeito dos mercados em que estão atuando (SPYROU, 2005; DEBASISH, 2009). Conforme Urso (2007), estes agentes, denominados de noise traders, têm dificuldade na interpretação das oscilações nos preços, o que faz com que assumam posições de acordo com a tendência observada nas cotações, provocando exacerbação do movimento e consequente afastamento do valor justo do ativo. A elevada volatilidade nos preços futuros é, então, transmitida às cotações spot, dado que são estritamente ligados pela possibilidade de arbitragem. Neste contexto, defende-se maior regulação nestes mercados, visto que a ação dos especuladores torna o sistema econômico mais susceptível a crises sistêmicas. Outros autores argumentam, no entanto, que este aumento da volatilidade, com a introdução dos derivativos, está relacionado ao fato de os mercados futuros e de opções viabilizarem a chegada de maior quantidade de informações, existindo maior velocidade de processamento e de transmissão destas últimas para 
o mercado spot (COX, 1976; GARCIA et al., 1986; ANTONIOU e HOLMES, 1995; ANTONIOU et al., 1998).

As pesquisas empíricas sobre tal tema se concentraram, em um primeiro momento, nos mercados futuros agropecuários e, a partir da década de 1970, voltaram-se aos derivativos financeiros (ações, títulos de renda fixa e moedas), dado o início a partir de então da negociação destes papéis nos mercados financeiros mundiais. No entanto, a recente alta dos preços das commodities na década de 2000 desperta o interesse novamente na análise destes produtos em um contexto em que se busca explicar as causas de tal movimento das cotações. Além dos fatores estruturais de oferta e demanda (consumo crescente de commodities observado, sobretudo, nos países do médio oriente; queda do nível de produção e dos estoques mundiais; elevação da oferta dos biocombustíveis) e de aspectos conjunturais (perda do poder de compra do dólar), aponta-se que este movimento altista pode também ser explicado pelo maior contágio dos derivativos agropecuários, de metais e de energia nos seus respectivos mercados à vista, em um processo conhecido como financeirização do mercado de commodities $^{5}$ (WRAY, 2009; REDRADO et al., 2009). Além de os movimentos especulativos com derivativos terem contribuído para tal alta, argumenta-se que esses papéis foram responsáveis pelo aumento da volatilidade das cotações à vista (UNCTAD, 2009).

No Brasil, derivativos sobre commodities agropecuárias são negociados na Bolsa de Valores, Mercadorias e Futuros (BM\&FBOVESPA) ${ }^{6}$. Na década de 2000, os papéis com maior volume de

5. Processo no qual agentes especuladores, como hedge funds, fundos de pensão e bancos de investimento, passam a utilizar, de forma intensa, os derivativos sobre commodities como ativo em seus portfólios (UNCTAD, 2009; REDRADO et al., 2009). Duas razões são apontadas para tal fato: i) a formação de preços das commodities é bem diferente da dos ativos tradicionais (como ações e títulos de renda fixa), o que leva a uma baixa correlação entre os retornos destes papéis, conferindo redução do risco da carteira; ii) tais derivativos são capazes de fornecer hedge contra a inflação (JENSEN et al., 2002).

6. Contratos de derivativos de café arábica, milho, soja, açúcar, etanol e boi gordo são negociados nesta bolsa. negociação no segmento agropecuário foram os contratos futuros de boi gordo e café arábica - em 2010 , responderam por $43,25 \%$ e $24,02 \%$, respectivamente, do total de contratos transacionados. Um significativo aumento das negociações foi observado nestes dois mercados no período destacado, especialmente na segunda metade desta década - enquanto que, em 2000, 147,5 mil e 386,9 mil contratos futuros de boi e café foram transacionados, respectivamente; em 2010, os números saltaram para 1,15 milhão e 640,76 mil contratos, nessa mesma ordem. Ao serem analisadas as safras brasileiras de café arábica dos anos 2000, verifica-se que as transações realizadas nos mercados futuros deste produto na BM\&FBOVESPA corresponderam, em média, a 2,1 safras nacionais - com destaque para o ano de 2007, quando o volume negociado foi equivalente a 3,2 safras. Já no mercado de boi gordo, a negociação com contratos futuros correspondeu, neste período, a $78 \%$ do volume abatido de bois no País, em média. Cabe observar ainda que, a partir de 2007, a bolsa brasileira apresentou negociações que superaram o número de cabeças de boi abatidas - em 2008, por exemplo, o número de contratos futuros transacionados foi equivalente a 2,3 vezes o total de bois abatidos em território nacional.

Diante dos cenários descritos acima - alta dos preços das commodities e elevação da liquidez das negociações com derivativos agropecuários no Brasil, este trabalho tem o objetivo de avaliar se este aumento da negociação dos contratos futuros de boi gordo e de café, transacionados na BM\&FBOVESPA, teve impacto na volatilidade das cotações spot durante a década de 2000.

De forma a atingir tal objetivo, testes de causalidade no sentido de Granger são empregados para avaliar os reflexos das alterações do volume de contratos futuros negociados de boi gordo e café, assim como do volume de contratos em aberto, sobre a volatilidade dos preços à vista dos respectivos mercados. Com aumento dos negócios com derivativos agropecuários na bolsa, ao admitir maior presença de noise traders nestas negociações e elevação da quantidade de informações transmitidas entre os mercados futuros 
e spot, espera-se que alterações no volume de negociação impactem as oscilações das cotações à vista. Alternativamente, considera-se também uma avaliação por meio de testes de causalidade entre a volatilidade dos preços futuros e a volatilidade dos preços à vista para os mercados de boi gordo e café arábica. Tais testes são baseados na função de correlação cruzada dos resíduos de modelos de volatilidade e no conceito do multiplicador de Lagrange. Nesse caso, a variabilidade nos preços assume papel central nessa discussão, em que se espera uma relação de bicausalidade entre a variação das cotações spot e das cotações futuras, como resultado do processo de formação dos preços futuros e da possibilidade de arbitragem entre os mercados à vista e de futuros.

$\mathrm{O}$ artigo está dividido em quatro partes, além desta introdução. Na seção 2, realiza-se uma revisão de literatura acerca do tema da pesquisa. Na terceira seção, apresenta-se a metodologia empregada neste trabalho. Em seguida, na seção 4 , os resultados são analisados e, por fim, na quinta seção, estão as conclusões e sugestões para trabalhos futuros.

\section{Revisão de literatura}

A influência dos mercados de derivativos sobre os preços spot de certo ativo consiste em uma análise essencialmente empírica. Observam-se quatro técnicas distintas: i) cômputo da volatilidade dos preços à vista do ativo antes e depois da criação do derivativo (com o uso de modelos da família $\mathrm{ARCH} / \mathrm{GARCH}^{7}$ ); ii) análise da volatilidade de ações inclusas e não inclusas em índice que passou a ter futuros em negociação; iii) avaliação da causalidade entre volume de contratos negociados e a volatilidade dos preços spot (MAYHEW, 2000; ILLUECA e LAFUENTE, 2003, 2008); iv) observação de causalidade entre as volatilidades dos preços futuros e

7. Proposto por Bollerslev (1986), o modelo Generalized Autoregressive Conditional Heteroscedasticity (GARCH) é uma generalização do Autoregressive Conditional Heteroscedasticity (ARCH) de Engle (1982). spot. Os resultados das pesquisas são contraditórios, dado que dependem do mercado de análise, do período estudado e da técnica utilizada.

A maior parte dos estudos tem base no mercado de índices de ações. Trabalhos recentes realizados em mercados de diferentes países apontam, em grande medida, que as negociações com derivativos não desestabilizaram as cotações à vista. Exemplos disso são Dawson e Staikouras (2009), Debasish (2009), Tripathy et al. (2009), Srinivasan e Bhat (2008), Kasman e Kasman (2008), Alexakis (2007), Drimbetas et al. (2007), Spyrou (2005), Bandivadekar e Ghosh (2003), Illueca e Lafuente (2003), Pilar e Rafael (2002), Bologna e Cavallo (2002) e Board et al. (2001). Por outro lado, Bae et al. (2009), Rao (2007), Zhong et al. (2004), Pok e Poshakwale (2004), Ryoo e Smith (2004) e Ahmad, Shah e Shah (2010) encontraram evidências de aumento na volatilidade das ações ou índices após a introdução dos contratos futuros ${ }^{8}$. Os mercados de títulos de renda fixa também foram objeto de análise nos estudos de Staikouras (2006), Hegde (1994) Moriarty e Tosini (1985) e Figlewski (1981), bem como o mercado de moedas nas pesquisas de Clifton (1985), Chatrath et al. (1996), Adrangi e Chatrath (1998), Brown e Cursi (2002), Jochum e Kodres (1998), Shastri et al. (1996) e Galvão et al. (2000).

Em relação às commodities, Mayhew (2000) lista as pesquisas feitas em diversos mercados, sendo que, em grande parte dos casos, houve um pequeno impacto nos preços à vista com a introdução da negociação dos derivativos - Tabela 1.

Poucos trabalhos recentes sobre o tema em questão foram realizados com commodities. Thraen (1998) analisou o mercado de queijo, concluindo que o mercado futuro de cheddar cheese da Chicago Mercantile Exchange (CME) não levou a um aumento da volatilidade do preço spot. Morgan (1999) observou que a introdução de futuros de batata no mercado londrino levou à redução da volatilidade dos preços desta com-

8. Para trabalhos anteriores à década de 2000, Mayhew (2000) realizou ampla revisão de literatura sobre este tema, constatando maior número de pesquisas com resultados similares aos do primeiro grupo de autores. 
Tabela 1. Trabalhos com análise do impacto na volatilidade dos preços spot a partir do início das negociações de contratos futuros sobre commodities

\begin{tabular}{l|l|l}
\hline \multicolumn{1}{c|}{ Autor } & \multicolumn{1}{|c|}{ Mercado(s), período e país de análise } & \multicolumn{1}{c}{ Resultado } \\
\hline Emery (1896) & Algodão (1821 a 1894) e trigo (1865 a 1895) / EUA & Estatística descritiva \\
Hooker (1901) & Trigo (1897 a 1899) / Alemanha, EUA e Inglaterra & Estatística descritiva \\
Working (1960) & Cebola (1946 a 1958) / EUA & Estatística descritiva \\
Gray (1963) & Cebola (1922 a 1962) / EUA & Estatística descritiva \\
Powers (1970) & Barriga de porco e boi gordo (1961 a 168) / EUA & Estatística descritiva e econometria de séries temporais \\
Tomek (1971) & Trigo (1841 a 1921) / EUA & Estatística descritiva \\
Johnson (1973) & Cebola (1930 a 1968) / EUA & Estatística descritiva e análise de regressão \\
Taylor e Leuthold (1974) & Boi gordo (1957 a 1972) / EUA & Estatística descritiva \\
Brorsen et al. (1989) & Boi gordo (1957 a 1982) / EUA & Econometria de séries temporais \\
Weaver e Banerjee (1990) & Boi gordo (1972 a 1981) / EUA & Econometria de séries temporais \\
Antoniou e Foster (1992) & Petróleo (1986 a 1990) / EUA & Modelo de volatilidade condicional autorregressiva \\
Netz (1995) & Trigo (1858-1865) / EUA & Sem impacto \\
Kocagil (1997) & Metais (1980-1990) / EUA & Bálise de regressão de modelo de estocagem \\
\hline
\end{tabular}

Fonte: Adaptado de Mayhew (2000).

modity. No entanto, três outros importantes trabalhos apontam para conclusões contrárias. Malliaris e Urrutia (1998) verificaram bicausalidade entre volatilidade dos preços e volume negociado nos mercados futuros norte-americanos de milho, soja em grão, farelo e óleo de soja. Kocagil e Shachmurove (1998) constataram causalidade bidirecional entre variação absoluta dos preços e volume de negociação de contratos futuros de milho, soja e açúcar. Porém, ao trabalhar com variação dos preços ao invés de variação absoluta, a causalidade foi unidirecional no sentido da variação dos preços para o volume negociado. Já Yang et al. (2005) analisaram os mercados norte-americanos de milho, soja, açúcar, trigo, algodão, boi gordo e suíno, observando, em geral, causalidade unidirecional no sentido de que um aumento não esperado do volume negociado de futuros causa aumento da volatilidade dos preços à vista.

\section{Metodologia}

\subsection{Dados}

Os dados utilizados neste estudo são: i) preços futuros de primeiro vencimento do boi gordo e café arábica9', divulgados pela BM\&FBOVESPA; ii) volume de contratos futuros negociados e em aberto; iii) indicadores para o mercado à vista destes ativos, calculados pelo CEPEA/USP/ESALQ ${ }^{10}$. O período de análise para o mercado de café se dá entre janeiro de 2000 e dezembro de 2010 e, para o mercado de boi gordo, entre janeiro de $2001^{11}$ e dezembro de 2010. Os Anexos 1 e 2 apresentam a evolução dos preços e das negociações.

\subsection{Análise da influência do mercado futuro sobre a volatilidade dos preços spot}

A fim de analisar o impacto dos contratos futuros sobre a volatilidade dos preços à vista nos mercados de café arábica e boi gordo, o presente

9. No mercado de boi, foi considerado o primeiro vencimento corrido. Já no mercado de café, foram utilizados os vencimentos de setembro e dezembro, dada a ampla liquidez destes papéis frente aos demais. Vale ainda mencionar que, neste último contrato, como não existia liquidação financeira, foram computados preços até um mês antes da data de vencimento (em período anterior à data de aviso de entrega).

10. Centro de Estudos Avançados em Economia Aplicada (Cepea) da Escola Superior de Agricultura "Luiz de Queiroz" (Esalq), Universidade de São Paulo (USP).

11. O início em janeiro de 2001 se deve às modificações do contrato de boi, que passou a ter unidade de cotação em R\$/@ (ao invés de US\$/@), além de admitir a possibilidade de liquidação financeira no vencimento. 
estudo empregará dois métodos. O primeiro tem base na análise de causalidade entre: i) contratos negociados e volatilidade dos preços spot; ii) contratos em aberto e volatilidade dos preços spot. $\mathrm{O}$ segundo consiste em dois testes de causalidade entre as volatilidades dos preços futuros e à vista, propostos por Cheung e Ng (1996) e Hafner e Herwartz (2006).

As volatilidades dos preços spot e futuros serão calculadas mediante um processo GARCH $(1,1)$, procedimento bastante comum nos trabalhos que versam sobre o tema (YANG et al., 2005). Os testes estatísticos mencionados acima são apresentados detalhadamente a seguir.

\subsubsection{Teste de causalidade \\ entre volume de negócios e volatilidade dos preços spot}

O primeiro método tem base em Yang et al. (2005), sendo feito em duas etapas. A primeira consiste no cálculo da volatilidade dos preços à vista. Este será realizado a partir de um modelo $\operatorname{GARCH}(1,1)$ :

$$
\mathrm{h}_{\mathrm{t}}=\alpha_{0}+\alpha_{1} \varepsilon_{\mathrm{t}-1}^{2}+\beta_{1} \mathrm{~h}_{\mathrm{t}-1}
$$

na qual $\alpha_{1}$ mede a extensão em que um choque no retorno em $t$ afeta a volatilidade em $t+1$. A soma $\left(\alpha_{1}+\beta_{1}\right)$ revela a medida de persistência da volatilidade, ou seja, a taxa que reflete como o impacto de um choque no retorno hoje se propaga ao longo do tempo sobre a volatilidade dos retornos futuros.

A segunda etapa é baseada no teste de causalidade entre a volatilidade dos preços à vista (Volspot) e os contratos negociados e em aberto. O objetivo é observar se mudanças em uma variável precedem alterações na outra variável ou se tais mudanças ocorrem simultaneamente. No entanto, de acordo com Bessembinder, Chan e Seguin (1996), para avaliar o impacto da negociação de derivativos sobre a volatilidade dos preços à vista, somente o componente não esperado dos contratos negociados (CNECN) deve ser considerado, dado que somente este pode ter influ- ência na variabilidade dos preços ${ }^{12}$. Para obtê-lo, será calculada a diferença entre o volume atual de negociação e uma média móvel do número de contratos transacionados nos 21 dias úteis anteriores. A partir destas duas variáveis, VolSpot e CNECN, será realizado o teste de causalidade de Granger ${ }^{13}$ - equações (2) e (3). A mesma análise será realizada entre VolSpot e o componente não esperado dos contratos em aberto (CNECA). Enquanto a variável CNECN se constitui em uma proxy para a atuação dos especuladores, o CNECA busca captar a atividade dos hedgers. Peck (1979), Garcia et al. (1986) e Malliaris e Urrutia (1998) mencionam que a variação no volume de negócios se constitui em proxy da atividade especulativa, dado que este tipo de operação se observa com mais frequência nas transações diárias. Existe menor participação dos negócios feitos por hedgers no volume total diário. Estes últimos tendem a manter a sua posição por um período maior de tempo; sendo assim, as variações dos contratos em aberto tendem a refletir a sua atuação.

$$
\begin{aligned}
& \text { VolSpot }_{t}=\theta_{0}+\sum_{i=1}^{p} a_{i} \text { VolSpot }_{t-1}+ \\
& +\sum_{j=1}^{q} b_{j} \text { CNECN }_{t-j}+u_{t}, \\
& \text { CNECN }_{t}=\gamma_{0}+\sum_{i=1}^{p} c_{i} \text { CNECN }_{t-1}+ \\
& +\sum_{j=1}^{q} d_{j} \text { VolSpot }_{t-j}+v_{t}
\end{aligned}
$$

em que $u_{\mathrm{t}} \mathrm{e} v_{\mathrm{t}}$ são termos aleatórios não correlacionados, $a_{\mathrm{i}}, b_{\mathrm{j}}, c_{\mathrm{i}}$, e $d_{\mathrm{j}}, i=0, \ldots, p$ e $j=1, \ldots, q$ são os parâmetros do modelo, sendo $p$ e $q$ as defasagens. Será usada, de forma arbitrária, defasagem máxima igual a 20 (dado que os dados são diários e em dias úteis).

A partir das estimações das equações (2) e (3), serão testadas as seguintes hipóteses nulas mediante o teste de Wald com restrição de coeficientes:

12. Por hipótese e seguindo a Teoria das Expectativas Racionais, as informações contidas no componente esperado do volume de contratos negociados já estão refletidas nos preços à vista.

13. Um passo anterior ao teste de causalidade de Granger consistiu no estudo da ordem de integração das variáveis a partir de testes de raízes unitárias. 
- $H_{0}: b_{1}=b_{2}=\ldots=b_{\mathrm{j}}=0$ - em que se conclui que $C N E C N_{\mathrm{t}}$ não Granger causa VolSpott.

- $H_{0}: d_{1}=d_{2}=\ldots=d_{\mathrm{j}}=0$-em que se conclui que VolSPott não Granger causa CNECN .

- $H_{0}: d_{1}=d_{2}=\ldots=d_{\mathrm{j}}=0$ e $b_{1}=b_{2}=\ldots=b_{\mathrm{j}}$ $=0-$ em que se conclui que VolSPott não Granger causa $C N E C N_{\mathrm{t}}$ e vice-versa.

Adicionalmente, verificar-se-á a decomposição da variância do erro de previsão, como realizado em Yang et al. (2005). Tal análise possibilitará observar a intensidade da relação causal entre as variáveis, constatando a parcela que cada variável endógena tem nos erros de previsão da variável dependente. Para tanto, será estimado um modelo autorregressivo vetorial, $\operatorname{VAR}(p)$, que consiste em um sistema de equações lineares dinâmicas, em que cada variável endógena é determinada por uma combinação linear de suas defasagens e das defasagens das variáveis endógenas de outras equações. Tendo as variáveis o mesmo número de defasagens $p, \mathrm{o} \operatorname{VAR}(p)$ pode ser representado por:

$$
\mathrm{Z}_{\mathrm{t}}=\mathrm{A}_{0}+\mathrm{A}_{1} \mathrm{Z}_{\mathrm{t}-1}+\ldots+\mathrm{A}_{\mathrm{p}} \mathrm{Z}_{\mathrm{t}-\mathrm{p}}+\vartheta_{\mathrm{t}}
$$

em que $Z_{\mathrm{t}}$ é um vetor $(n \times 1)$ que inclui as $n$ variáveis, $A_{\mathrm{i}}$, com $i=0,1, \ldots, p$, é a matriz dos coeficientes, e $\vartheta_{\mathrm{t}} \approx \operatorname{IN}\left(0, \Sigma_{\mathrm{t}}\right)$ é o vetor das inovações do processo com distribuição Gaussiana multivariada de média zero e matriz de variância-covariância não singular $\Sigma_{\mathrm{t}}{ }^{14}$.

Os modelos VAR permitem capturar o efeito dinâmico das inter-relações das variáveis, tendo caráter simultâneo. A estimação é feita pelo método de Mínimos Quadrados Ordinários (MQO) em três estágios, em que: i) as equações são estimadas separadamente utilizando MQO, ii) os resíduos do passo anterior são utilizados para estimar as variâncias e covariâncias dos erros, e iii) essas estimativas das variâncias dos erros são usadas para estimar as equações conjuntamente.

Estimado o VAR, para explorar melhor as relações entre variáveis, será utilizado o modelo

14. Esse processo garante que os erros sejam i.i.d, indicando ausência de correlação serial. de decomposição da variância dos erros de previsão. Considerando-se ainda $Z_{t}$ o vetor das variáveis em questão, seguindo um processo de médias móveis infinito, tem-se:

$$
\mathrm{Zt}=\sum_{\mathrm{i}}^{\infty} \mathrm{B}_{\mathrm{i}} \zeta_{\mathrm{t}-1}, \mathrm{t}=1,2, \ldots, \mathrm{T},
$$

em que $B$ é a matriz dos parâmetros e $\zeta_{t}$ representa um processo ruído branco.

A generalização da decomposição da variância dos erros de previsão do vetor $Z_{t}$, seguindo Yang et al. (2005), é tal que:

$$
\phi_{i j}(n)=\frac{\sigma_{i i}^{-1} \sum_{l=1}^{n}\left(e_{i}^{\prime} B_{1} \Sigma e_{j}\right)^{2}}{\sum_{l=1}^{n}\left(e_{i}^{\prime} B_{1} \Sigma B_{i}^{\prime} e_{i}\right)}, i, j=1,2, \ldots, m,
$$

em que $\sigma_{\mathrm{ii}}$ é o elemento $i i$ da matriz de variância-covariância $\Sigma$ dos resíduos do vetor $Z_{\mathrm{t}}, e_{\mathrm{j}}$ é um vetor que associa o valor 1 à linha $j$ e aos demais elementos o valor zero e $n$ consiste no número de passos à frente na previsão (YANG et al., 2005). A partir deste método, identifica-se qual porcentagem da variância dos erros de previsão pode ser atribuída aos choques não antecipados da própria variável e das demais variáveis endógenas do sistema, de forma separada.

\subsubsection{Teste de causalidade na volatilidade}

De forma a reforçar a análise da seção anterior, serão realizados testes de causalidade na variância dos preços, desenvolvidos por Cheung e Ng (1996) e Hafner e Herwartz (2006). Tais testes serão feitos a partir dos preços obtidos entre 2007 e 2010, quando o volume de negociação nos mercados em questão teve crescimento significativo ${ }^{15}$.

O método de Cheung e Ng (1996) se baseia na análise da função de correlação cruzada (FCC) dos quadrados dos resíduos padronizados. O procedimento é realizado em dois estágios: i) estima-

15. No mercado futuro de boi, enquanto entre 2001-2006 verificou-se média de cerca de 210 mil contratos negociados por ano, entre 2007 e 2010, tal volume passa para mais de um milhão de contratos. Já no mercado futuro de café, verifica-se aumento de aproximadamente 480 mil contratos negociados por ano entre 2000 e 2006 para 650 mil entre 2007 e 2010. 
424 - Derivativos sobre Commodities Influenciam a Volatilidade dos Preços à Vista? Uma análise nos mercados de boi gordo e café arábica no Brasil

ção de modelos GARCH univariados; ii) obtenção da série dos quadrados dos resíduos padronizados $u_{\mathrm{t}}$ e $v_{\mathrm{t}}$ das séries dos preços spot e futuro, $X_{\mathrm{t}}$ e $Y_{\mathrm{t}}$, respectivamente, proveniente da variância condicional - equações (7) e (8).

$$
\begin{aligned}
& u_{t}=\frac{\left(X_{t}-\mu_{x, t}\right)^{2}}{h_{x, t}}=\xi_{1 t}^{2}, \\
& v_{t}=\frac{\left(Y_{t}-\mu_{y, t}\right)^{2}}{h_{y, t}}=\xi_{2 t}^{2},
\end{aligned}
$$

em que $\mu_{x, t}$ e $\mu_{y, t}$ correspondem aos valores médios das séries de mercado spot e futuro, respectivamente, e $h_{\mathrm{x}, \mathrm{t}}$ e $h_{\mathrm{Y}, \mathrm{t}}$ consistem nas variâncias condicionais. A correlação cruzada no instante $k, r_{\mathrm{uv}}(\mathrm{k})$ é dada por:

$$
\mathrm{r}_{\mathrm{uv}}=\mathrm{c}_{\mathrm{uv}}(\mathrm{k})\left(\mathrm{c}_{\mathrm{uu}}(0) \mathrm{c}_{\mathrm{vv}}(0)\right)^{-1 / 2}
$$

na qual $\quad \mathrm{c}_{\mathrm{uv}}(\mathrm{k})=\mathrm{N}^{-1} \sum_{\mathrm{t}=1}^{\mathrm{N}}\left(\mathrm{u}_{\mathrm{t}}-\overline{\mathrm{u}}\right)\left(\mathrm{v}_{\mathrm{t}}-\overline{\mathrm{v}}\right)$; $\mathrm{k}=0, \pm 1, \pm 2, \ldots, \mathrm{c}_{\mathrm{uu}}(0)$ e $\mathrm{c}_{\mathrm{vv}}(0)$ são as variâncias de $u_{\mathrm{t}}$ e $v_{\mathrm{t}}$, respectivamente, e $T$ é o tamanho da amostra.

A partir das estimações, utiliza-se a FCC de $u$ e $v$ para testar a hipótese nula de não causalidade na variância. Avalia-se a significância da FCC de forma a detectar relações causais e identificar a direção da causalidade. Para o teste de causalidade no instante $k$, compara-se $\sqrt{\operatorname{Tr}_{\mathrm{uv}}(\mathrm{k})}$ com uma distribuição normal padronizada.

De forma complementar, aplicar-se-á a metodologia proposta por Hafner e Herwartz (2006), a qual tem base no princípio do Multiplicador de Lagrange (ML). A partir de um processo estocástico $\left\{\varepsilon_{\mathrm{t}} \in \mathbb{R}^{\mathrm{n}}, \mathrm{t} \in \mathbb{N}\right\}$ estacionário, no espaço de probabilidade $(\Omega, F, P)$, em que $\mathrm{E}\left(\varepsilon_{\mathrm{it}} \mid \mathrm{F}_{\mathrm{t}-1}\right)=0$ , testa-se a hipótese nula, $H_{0}$, para um dado $i, j=$ $1, \ldots, n, i \neq j$ :

$$
\mathrm{H}_{0}: \operatorname{Var}\left(\varepsilon_{\mathrm{it}} \mid \mathrm{F}_{\mathrm{t}-1}^{(\mathrm{j})}\right)=\operatorname{Var}\left(\varepsilon_{\mathrm{it}} \mid \mathrm{F}_{\mathrm{t}-1}\right),
$$

sendo $\mathrm{F}_{\mathrm{t}-1}^{(\mathrm{j})}=\mathrm{F}_{\mathrm{t}} \backslash \mathrm{h}\left(\varepsilon_{\mathrm{j},}, \tau \leq \mathrm{t}\right)$.

A hipótese nula implica não causalidade na variância. Neste sentido, as informações da variância de outros processos estocásticos não afetam a variância do processo em questão. Para proceder ao teste, considera-se:

$$
\begin{aligned}
& \varepsilon_{\mathrm{it}}=\xi_{\mathrm{it}} \sqrt{\mathrm{h}_{\mathrm{it}}^{2} \mathrm{~g}_{\mathrm{t}}}, \mathrm{g}_{\mathrm{t}}=1+\mathrm{z}_{\mathrm{jt}}^{\mathrm{T}} \pi, \\
& \mathrm{z}_{\mathrm{jt}}=\left(\varepsilon_{\mathrm{jt}-1}^{2}, \mathrm{~h}_{\mathrm{jt}-1}^{2}\right)^{\mathrm{T}},
\end{aligned}
$$

em que $\xi_{\text {it }}$ é o resíduo padronizado dado pelas equações(7)e (8)e $\operatorname{Var}\left(\varepsilon_{\mathrm{it}} \mid \mathrm{F}_{\mathrm{t}-1}\right)=\alpha_{0}+\alpha_{\mathrm{i}} \varepsilon_{\mathrm{i}, \mathrm{t}-1}^{2}+\beta_{\mathrm{i}} \mathrm{h}_{\mathrm{it}-1}^{2}$. Pela última expressão, nota-se que o método utiliza um GARCH $(1,1)$.

Com base na equação (11), verifica-se que $\pi=$ 0 é uma condição suficiente para que a hipótese nula seja verdadeira (HAFNER e HERWARTZ, 2006). Assim sendo, as hipóteses podem ser reformuladas, de tal forma que $H_{0}: \pi=0$ e $H_{1}: \pi \neq 0$.

A estatística $\lambda_{\mathrm{LM}}{ }^{16}$ é utilizada para realização do teste. Três etapas são utilizadas para obtê-la: i) estimação do $\operatorname{GARCH}(1,1)$ e consequente obtenção dos resíduos padronizados $\xi_{\text {it, }}$ das derivadas $x_{\mathrm{it}}$ e da volatilidade $h_{\mathrm{it}}^{2}$; ii) regressão de $\xi_{\mathrm{it}}^{2}-1$ contra $x_{\mathrm{it}}^{\mathrm{T}}$; iii) cálculo da estatística $\lambda_{\mathrm{LM}}$ mediante a multiplicação entre $N$ e o coeficiente de determinação $\left(R^{2}\right)$ da regressão no passo anterior. Caso o $\lambda_{\mathrm{LM}}$ calculado supere o valor crítico (obtido a partir de uma distribuição $\chi_{2}^{2}$ ), rejeita-se a hipótese nula de não causalidade na variância.

\section{Análise dos resultados}

\subsection{Causalidade entre volume de negócios e volatilidade dos preços spot}

A Tabela 2 apresenta as estatísticas descritivas das séries de volatilidade dos preços à vista (VolSpot), obtida a partir de um GARCH $(1,1)$, e do componente não esperado do volume de contratos negociados (CNECN) e em aberto (CNECA). Os Anexos 3 e 4 apresentam os gráficos da evolução da volatilidade de tais séries.

$$
\begin{aligned}
& \text { 16. } \lambda_{\mathrm{ML}}=\frac{1}{4 \mathrm{~T}}\left[\sum_{\mathrm{t}=1}^{\mathrm{T}}\left(\zeta_{\mathrm{jt}}^{2}-1\right) \mathrm{Z}_{\mathrm{jt}}^{\mathrm{t}}\right] \mathrm{V}\left(\theta_{\mathrm{i}}\right)^{-1}\left[\sum_{\mathrm{t}=1}^{\mathrm{T}}\left(\zeta_{\mathrm{jt}}^{2}-1\right) \mathrm{Z}_{\mathrm{jt}}\right] \\
& \text { sendo: } \\
& \mathrm{V}\left(\theta_{\mathrm{i}}\right)=\frac{\mathrm{k}}{4 \mathrm{~N}}\left[\sum_{\mathrm{t}=1}^{\mathrm{N}} \mathrm{z}_{\mathrm{jt}} \mathrm{Z}_{\mathrm{jt}}^{\mathrm{T}}-\sum_{\mathrm{t}=1}^{\mathrm{N}} \mathrm{z}_{\mathrm{jt}} \mathrm{x}_{\mathrm{jt}}^{\mathrm{T}}\left(\sum_{\mathrm{t}=1}^{\mathrm{N}} \mathrm{x}_{\mathrm{jt}} \mathrm{x}_{\mathrm{jt}}^{\mathrm{T}}\right)^{-1}\right] \sum_{\mathrm{t}=1}^{\mathrm{N}} \mathrm{x}_{\mathrm{jt}} \mathrm{Z}_{\mathrm{jt}}^{\mathrm{T}}
\end{aligned}
$$

Em que:

$\mathrm{k}=\frac{1}{\mathrm{~N}} \sum_{\mathrm{t}=1}^{\mathrm{N}}\left(\xi_{\mathrm{it}}^{2}-1\right)^{2} ; \mathrm{x}_{\mathrm{it}}=\mathrm{h}_{\mathrm{it}}^{-2}\left(\partial \mathrm{h}_{\mathrm{it}}^{2} / \partial \theta_{\mathrm{i}}\right) ; \theta_{\mathrm{i}}=\left(\alpha_{0}, \alpha_{\mathrm{i}}, \beta_{\mathrm{i}}\right)^{\mathrm{T}} .$. 
Tabela 2. Estatísticas descritivas das séries de volatilidade dos preços spot e componente não esperado de contratos futuros negociados e em aberto de café e boi entre janeiro de 2000 e dezembro de 2010

\begin{tabular}{lcccccc}
\hline & \multicolumn{3}{c}{ Café Arábica } & \multicolumn{3}{c}{ Boi Gordo } \\
\cline { 2 - 7 } & VolSpot & CNECN & CNECA & VolSpot & CNECN & CNECA \\
\hline Média & 0,0187 & 2,8697 & 23,8072 & 0,000029 & 10,8800 & 40,5419 \\
Mediana & 0,0174 & $-159,3750$ & 72,9750 & 0,000013 & $-60,5000$ & 335,8500 \\
Máximo & 0,0906 & $15.205,40$ & $6.393,60$ & 0,000572 & $15.453,45$ & $1.4095,00$ \\
Mínimo & 0,0096 & $-3.410,60$ & $-7.383,65$ & 0,000002 & $-6.264,40$ & $-22.464,50$ \\
Desvio Padrão & 0,0073 & $1.259,20$ & $1.559,00$ & 0,000048 & $1.332,40$ & $3.090,17$ \\
Assimetria & 3,8199 & 1,7268 & $-0,2641$ & 4,683263 & 1,2598 & $-1,4840$ \\
Curtose $^{\text {Jarque-Bera }}{ }^{1}$ & 28,5632 & 14,0912 & 6,7080 & 34,420910 & 15,2585 & 12,7770 \\
\hline
\end{tabular}

${ }^{1}$ Para todas as séries avaliadas o teste Jarque-Bera indicou não normalidade.

Fonte: Resultados da pesquisa.

Tabela 3. Teste de causalidade de Granger entre as variáveis: volatilidade dos preços à vista (VolSpot) e componente não esperado de contratos futuros negociados $(C N E C N)$ de café e boi gordo

\begin{tabular}{|c|c|c|c|c|c|c|c|}
\hline \multirow{2}{*}{ Mercado } & \multirow{2}{*}{ Hipótese Nula } & \multicolumn{6}{|c|}{ Estatística F } \\
\hline & & $\operatorname{Lag} 1$ & $\operatorname{Lag} 2$ & $\operatorname{Lag} 5$ & $\operatorname{Lag} 10$ & $\operatorname{Lag} 15$ & $\operatorname{Lag} 20$ \\
\hline \multirow{2}{*}{ Café } & VolSpot não Granger Causa CNECN & $264,51^{* *}$ & $132,25^{* *}$ & $53,91^{* * *}$ & $27,12^{* *}$ & $17,8^{* *}$ & $13,76^{*}$ \\
\hline & CNECN não Granger Causa VolSpot & $5,63^{* * *}$ & $3,55^{* * *}$ & $4,46^{* * *}$ & $21,42^{* * *}$ & $16,82^{* * *}$ & $14,71^{* * *}$ \\
\hline \multirow{2}{*}{ Boi Gordo } & VolSpot não Granger Causa CNECN & $16,49^{* * *}$ & $8,29 * * *$ & $6,17^{* * *}$ & $3,66^{* * *}$ & $3,15^{* * *}$ & $3,06^{* * *}$ \\
\hline & CNECN não Granger Causa VolSpot & $5,70^{* * *}$ & $5,86^{* * *}$ & $4,90^{* * *}$ & $3,56^{* * *}$ & $2,83^{* * *}$ & $2,82^{* * *}$ \\
\hline
\end{tabular}

* Significativo a $10 \%$; ** Significativo a 5\%; *** Significativo a $1 \%$.

Fonte: Resultados da pesquisa.

Ao realizar os testes Phillips-Perron, foi verificado que as três séries são estacionárias ${ }^{17}$ Anexo 5. Tal conclusão também foi constatada nos estudos de Bessembinder e Seguim (1992), Bessembinder, Chan e Seguin (1996) e Yang et al. (2005).

A partir da constatação acima, foram feitos os testes de causalidade de Granger. Estes indicaram uma bicausalidade (no sentido de Granger) entre volatilidade dos preços spot (VolSpot) e componente não esperado do volume de contratos negociados $(C N E C N)$ nos mercados de café arábica e de boi gordo (Tabela 3$)^{18}$. Admitindo-se que as variações no volume de contratos negociados tendem a representar a atividade especulativa, os resultados permitem inferir que especulado-

17. Também foram feitos testes ADF (Augmented Dickey-Fuller), que indicaram estacionariedade em nível.

18. As estatísticas obtidas foram significativas e indicaram a rejeição da hipótese nula de não causalidade para os distintos lags avaliados (diferentes defasagens para as variáveis explicativas dos modelos). res atuantes nos mercados futuros de boi e café influenciaram o padrão da volatilidade dos preços à vista no período de análise. Conclusões similares foram obtidas por Malliaris e Urrutia (1998), que constataram causalidade bidirecional entre as variáveis em estudo. Já Yang et al. (2005) obtiveram bicausalidade apenas no mercado de açúcar.

Verificou-se também relação bicausal entre volatilidade das cotações à vista e componente não esperado do volume de contratos em aberto (CNECA) no mercado de boi, sinalizando que a atuação dos hedgers também exerceu influência nas oscilações dos preços spot do boi gordo (Tabela 4). Tal fato não ocorreu no mercado de café, em que houve ausência de causalidade nos dois sentidos. Os resultados de Yang et al. (2005) foram bastante diferentes neste ponto, dado que, em todos os contratos analisados, os autores observaram causalidade unidirecional (sendo o sentido da causalidade dependente do mercado analisado). 
426 - Derivativos sobre Commodities Influenciam a Volatilidade dos Preços à Vista? Uma análise nos mercados de boi gordo e café arábica no Brasil

Tabela 4. Teste de causalidade de Granger entre as variáveis: volatilidade dos preços à vista (VolSpot) e componente não esperado de contratos futuros em aberto (CNECA) de café e boi gordo

\begin{tabular}{clcccccc}
\hline \multirow{2}{*}{ Mercado } & \multirow{2}{*}{ Hipótese Nula } & \multicolumn{5}{c}{ Estatística F } \\
\cline { 3 - 8 } & & $\operatorname{Lag} 1$ & $\operatorname{Lag} 2$ & $\operatorname{Lag} 5$ & $\operatorname{Lag} 10$ & $\operatorname{Lag} 15$ & $\operatorname{Lag} 20$ \\
\hline \multirow{2}{*}{ Café } & VolSpot não Granger Causa CNECA & 0,02 & 1,06 & 1,26 & 1,02 & 1,07 & 0,87 \\
& CNECA não Granger Causa VolSpot & 0,04 & 0,37 & 0,42 & 0,47 & 0,58 & 0,58 \\
\hline \multirow{2}{*}{ Boi Gordo } & VolSpot não Granger Causa CNECA & $5,95^{* *}$ & $4,29^{* *}$ & $2,47^{* *}$ & $1,90^{* *}$ & 1,24 & 1,15 \\
& CNECA não Granger Causa VolSpot & $7,83^{* * *}$ & $5,91^{* * *}$ & $2,50^{* *}$ & $1,73^{*}$ & $1,99^{* *}$ & $2,11^{* * *}$ \\
\hline
\end{tabular}

* Significativo a $10 \%$; ** Significativo a 5\%; *** Significativo a $1 \%$.

Fonte: Resultados da pesquisa.

Tabela 5. Decomposição da variância do erro de previsão - análise bivariada: VolSpot e CNECN

\begin{tabular}{l|c|cc|cc}
\hline \multirow{2}{*}{ Mercado } & \multirow{2}{*}{ Dia } & \multicolumn{2}{|c}{ VolSpot explicada por } & \multicolumn{2}{c}{ CNECN explicada por } \\
\cline { 2 - 5 } & & VolSpot & CNECN & VolSpot & CNECN \\
\hline \multirow{3}{*}{ Café } & 1 & 100,00 & 0,00 & 0,18 & 99,82 \\
Arábica & 5 & 93,87 & 6,13 & 0,49 & 99,51 \\
& 10 & 93,75 & 6,25 & 0,55 & 99,45 \\
& 15 & 93,78 & 6,22 & 0,83 & 99,17 \\
\hline \multirow{4}{*}{ Boi } & 20 & 93,81 & 6,19 & 1,10 & 98,90 \\
Gordo & 1 & 100,00 & 0,00 & 0,24 & 99,76 \\
& 5 & 99,29 & 0,70 & 0,47 & 99,53 \\
& 10 & 98,88 & 1,12 & 1,65 & 98,35 \\
\hline
\end{tabular}

Fonte: Resultados da pesquisa.

\subsubsection{Decomposição do erro de previsão - análise bivariada}

Após a execução dos testes de causalidade de Granger, foi feita a decomposição da variância do erro de previsão a fim de avaliar a intensidade da relação causal observada na seção anterior. Em um primeiro momento, considerou-se um VAR a partir de dois pares de variáveis: VolSpot e CNECN; VolSpot e CNECA ${ }^{19}$.

A Tabela 5 mostra os resultados da decomposição da variância do erro de previsão para cinco

19. No mercado de café, a partir dos critérios de informação SC (Schwarz Information Criterion) e HQ (Hannan-Quinn Information Criterion), houve convergência para um VAR de ordem igual a cinco para os dois pares de variáveis em análise. A ordem de entrada das variáveis no modelo VAR (da mais exógena para a mais endógena) não influenciou nos resultados. Já, para o mercado de boi gordo, a ordem do VAR foi igual a cinco para o primeiro par de variáveis (VolSpot e CNECN), adotando-se o menor número de defasagens entre os critérios considerados, e igual a um para o segundo par (VolSpot e CNECA) - Anexo 6. Como no caso do mercado de café, a ordem de entrada das variáveis no modelo não interferiu nos resultados. períodos de tempo dentro de 21 dias úteis. No mercado de café, os erros de previsão da volatilidade dos preços no mercado à vista (VolSpot) foram atribuídos, em aproximadamente 94\%, à própria volatilidade e, cerca de $6 \%$, ao componente não esperado do volume negociado (CNECN). Ou seja, CNECN influenciou VolSpot, confirmando uma possível interferência da atividade especulativa, presente nos mercados futuros, sobre as oscilações das cotações spot. Tal resultado é consistente com a causalidade de Granger. Por outro lado, VolSpot levou a pouca variação em CNECN. No mercado de boi, por sua vez, não se observou influência significativa entre as variáveis - ou seja, a relação causal entre as variáveis teve pequena intensidade.

Nos modelos VAR compostos pelas variáveis VolSpot e componente não esperado dos contratos em aberto (CNECA), os erros de previsão foram, em boa parte, explicados pela própria variável, conforme mostra a Tabela 6. Assim, a relação bicausal entre CNECA e VolSpot observada no 
Tabela 6. Decomposição da variância do erro de previsão - análise bivariada: VolSpot e CNECA

\begin{tabular}{|c|c|c|c|c|c|}
\hline \multirow{2}{*}{ Mercado } & \multirow{2}{*}{ Dia } & \multicolumn{2}{|c|}{ VolSpot explicada por } & \multicolumn{2}{|c|}{ CNECA explicada por } \\
\hline & & Volspot & CNECA & Volspot & CNECA \\
\hline \multirow{5}{*}{$\begin{array}{l}\text { Café } \\
\text { Arábica }\end{array}$} & 1 & 100,00 & 0,00 & 0,09 & 99,91 \\
\hline & 5 & 99,99 & 0,01 & 0,05 & 99,95 \\
\hline & 10 & 99,99 & 0,01 & 0,09 & 99,91 \\
\hline & 15 & 99,99 & 0,01 & 0,09 & 99,91 \\
\hline & 20 & 99,99 & 0,01 & 0,09 & 99,91 \\
\hline \multirow{5}{*}{$\begin{array}{l}\text { Boi } \\
\text { Gordo }\end{array}$} & 1 & 100,00 & 0,00 & 0,11 & 99,89 \\
\hline & 5 & 99,78 & 0,22 & 0,12 & 99,88 \\
\hline & 10 & 99,16 & 0,84 & 0,49 & 99,51 \\
\hline & 15 & 98,49 & 1,51 & 0,85 & 99,15 \\
\hline & 20 & 97,97 & 2,03 & 1,11 & 98,89 \\
\hline
\end{tabular}

Fonte: Resultados da pesquisa.

mercado de boi gordo, de acordo com os resultados da Tabela 4, apresentou baixa intensidade. Vale lembrar que, para o mercado de café, verificou-se ausência de relação causal entre estas variáveis, sendo já esperados os resultados apresentados na Tabela 6, i.e., os erros de previsão foram explicados essencialmente pelas próprias variáveis. Com isso, a atuação dos hedgers nos mercados futuros não estaria impactando a volatilidade presente no mercado à vista.

\subsubsection{Decomposição do erro de previsão - análise tri-variada}

Em uma segunda etapa, conforme realizado em Yang et al. (1995), incluiu-se no VAR a volatilidade dos preços futuros (VolFut ${ }^{20}$. As Tabelas $7 \mathrm{e}$ 8 apresentam os resultados da decomposição da variância do erro de previsão, considerando análise de dois conjuntos de dados compostos por três variáveis, respectivamente: VolSpot, VolFut e CNECN; e VolSpot, VolFut e CNECA. Nota-se que as inferências feitas a partir dos resultados do caso bivariado são próximas àquelas feitas para o caso tri-variado.

$\mathrm{Na}$ Tabela 7, verifica-se que volatilidade dos preços spot sofreu influência de CNECN (especialmente nos mercados de café, confirmando os

20. Os mesmos procedimentos de análise da ordem do VAR e do ordenamento das variáveis foram realizados, sendo os resultados similares aos da análise bivariada. resultados anteriores). Os erros de previsão da variável VolSpot no mercado de café arábica foram atribuídos em cerca de $90 \%$ à própria volatilidade, em aproximadamente $9 \%$ ao componente não esperado do volume negociado (CNECN) e próximo a $1 \%$ à volatilidade dos preços futuros. Já no mercado de boi gordo, os erros de previsão da volatilidade spot estão associados em aproximadamente $88 \%$ às próprias oscilações das cotações spot, $4 \%$ ao CNECN e $8 \%$ à volatilidade dos preços futuros ${ }^{21}$. Tais resultados dão ênfase no sentido de que os movimentos não esperados no volume negociado impactaram o padrão de volatilidade dos mercados em análise. Vale observar que os resultados de Yang et al. (2005) foram bastante similares.

A Tabela 8, por sua vez, indica que, nos dois mercados, a variável CNECA teve influência marginal sobre os erros de previsão de VolSpot, como obtido anteriormente com os pares de variáveis (análise bivariada). Assim, confirma-se que a atuação dos hedgers não exerceu influência significativa no padrão de oscilação dos preços em ambos os mercados.

21. A volatilidade spot levou a pouca variação de CNECN. A porcentagem de variação dos erros de previsão de $C N E C N$ no mercado de café foi explicada majoritariamente pela própria variável. Resultado similar foi obtido no mercado de boi gordo. A relação de causalidade de VolSpot para CNECN se mostrou relativamente frágil ao avaliar tais constatações. 

arábica no Brasil

Tabela 7. Decomposição da variância do erro de previsão, considerando-se as variáveis VolSpot, VolFut e CNECN

\begin{tabular}{c|c|ccc|ccc|ccc}
\hline \multirow{2}{*}{ Mercado } & \multirow{2}{*}{ Dia } & \multicolumn{3}{|c|}{ Volspot explicada por } & \multicolumn{3}{c|}{ VolFut explicada por } & \multicolumn{3}{c}{ CNECN explicada por } \\
\cline { 3 - 11 } & & VolSpot & VolFut & CNECN & VolSpot & VolFut & CNECN & VolSpot & VolFut & CNECN \\
\hline \multirow{3}{*}{ Café } & 1 & 100,00 & 0,00 & 0,00 & 39,17 & 60,82 & 0,00 & 0,22 & 0,33 & 99,43 \\
Arábica & 5 & 92,17 & 0,53 & 7,29 & 40,10 & 53,69 & 6,19 & 0,24 & 0,50 & 99,24 \\
& 10 & 90,77 & 0,57 & 8,64 & 42,77 & 49,64 & 7,58 & 0,31 & 0,72 & 98,96 \\
& 15 & 90,38 & 0,55 & 9,06 & 45,25 & 46,59 & 8,14 & 0,37 & 0,84 & 98,77 \\
& 20 & 90,22 & 0,52 & 9,25 & 47,35 & 44,18 & 8,46 & 0,42 & 0,91 & 98,65 \\
\hline \multirow{3}{*}{ Boi } & 1 & 100,00 & 0,00 & 0,00 & 0,64 & 99,35 & 0,00 & 0,38 & 0,04 & 99,57 \\
Gordo & 5 & 95,83 & 2,24 & 1,91 & 5,34 & 94,20 & 0,45 & 0,48 & 1,43 & 98,08 \\
& 10 & 88,47 & 8,15 & 3,36 & 12,56 & 85,74 & 1,68 & 0,77 & 2,86 & 96,35 \\
& 15 & 86,83 & 8,98 & 4,17 & 15,79 & 81,44 & 2,76 & 1,26 & 3,29 & 95,43 \\
& 20 & 85,85 & 9,49 & 4,64 & 18,41 & 78,02 & 3,55 & 1,54 & 3,64 & 94,80 \\
\hline
\end{tabular}

Fonte: Resultados da pesquisa.

Tabela 8. Decomposição da variância do erro de previsão, considerandos-se as variáveis VolSpot, VolFut e CNECA

\begin{tabular}{c|c|ccc|ccc|ccc}
\hline \multirow{2}{*}{ Mercado } & \multirow{2}{*}{ Dia } & \multicolumn{3}{|c|}{ Volspot explicada por } & \multicolumn{3}{c|}{ VolFut explicada por } & \multicolumn{3}{c}{ CNECA explicada por } \\
\cline { 3 - 10 } & & VolSpot & VolFut & CNECA & VolSpot & VolFut & CNECA & VolSpot & VolFut & CNECA \\
\hline \multirow{3}{*}{ Café } & 1 & 100,00 & 0,00 & 0,00 & 43,02 & 56,97 & 0,00 & 0,07 & 0,12 & 99,79 \\
Arábica & 5 & 99,53 & 0,42 & 0,03 & 46,60 & 53,36 & 0,03 & 0,01 & 0,29 & 99,68 \\
& 10 & 99,48 & 0,47 & 0,03 & 50,04 & 49,92 & 0,03 & 0,00 & 0,53 & 99,45 \\
& 15 & 99,47 & 0,48 & 0,03 & 52,88 & 47,08 & 0,03 & 0,01 & 0,77 & 99,20 \\
& 20 & 99,47 & 0,48 & 0,03 & 55,19 & 44,77 & 0,02 & 0,01 & 1,01 & 98,96 \\
\hline \multirow{3}{*}{ Boi } & 1 & 100,00 & 0,00 & 0,00 & 0,54 & 99,45 & 0,00 & 0,07 & 0,00 & 99,91 \\
Gordo & 5 & 97,29 & 2,64 & 0,06 & 5,44 & 94,29 & 0,25 & 0,15 & 2,48 & 97,36 \\
& 10 & 90,37 & 9,45 & 0,16 & 12,93 & 86,53 & 0,52 & 0,60 & 4,26 & 95,13 \\
& 15 & 88,53 & 10,95 & 0,50 & 16,00 & 83,56 & 0,42 & 0,70 & 4,94 & 94,34 \\
& 20 & 87,17 & 11,78 & 1,03 & 18,60 & 81,05 & 0,33 & 0,75 & 5,29 & 93,95 \\
\hline
\end{tabular}

Fonte: Resultados da pesquisa.

Em síntese, as análises até então desenvolvidas mostram importante contribuição de CNECN (proxy da atividade especulativa nos mercados) sobre a VolSpot no mercado de café arábica. Já no mercado de boi gordo, tal contribuição foi um pouco menor - no entanto, a volatilidade dos preços futuros (VolFut) teve importante influência sobre as oscilações dos preços à vista (VolSpot), sendo que esta primeira variável tem influência de CNECN. Por outro lado, a variável CNECA, que capta a movimentação dos hedgers, não exerceu influência sobre a volatilidade no mercado spot no mercado de café, sendo que, no mercado de boi, apesar da existência de causalidade, a intensidade de tal relação se mostrou marginal.

\subsection{Causalidade na volatilidade entre preços spot e preços futuros}

Avaliada a relação entre o volume de negócios e a volatilidade dos preços à vista, verificou-se a causalidade na variância, considerando-se as cotações à vista e futuras ${ }^{22}$. A Tabela 9 apresenta os resultados do teste de Cheung e Ng (1996). Para o mercado de boi gordo, é verificada causalidade bidirecional, uma vez que as estatísticas mostraram-se significativas nas defasagens posi-

22. Como descrito na metodologia, os testes levam em conta preços de janeiro de 2007 a dezembro de 2010, uma vez que se observa, neste período, forte aumento das negociações dos derivativos em questão. 
Tabela 9. Teste de causalidade de Cheung e Ng (1996) entre as volatilidades à vista e futura dos contratos de café e boi entre janeiro de 2007 e dezembro de 2010

\begin{tabular}{ccccc}
\hline \multirow{2}{*}{ Defasagem } & \multicolumn{2}{c}{ Café Arábica } & \multicolumn{2}{c}{ Boi Gordo } \\
\cline { 2 - 5 } & $r_{u v}(k)$ & $p$-valor & $r_{u v}(k)$ & 0,0312 \\
5 & $-0,0218$ & 0,7408 & $0,0628^{* *}$ & 0,0008 \\
3 & $-0,0202$ & 0,7431 & $0,1065^{* * *}$ & 0,0120 \\
2 & 0,0143 & 0,3356 & $0,0760^{* *}$ & 0,0000 \\
1 & $0,0512^{*}$ & 0,0641 & $0,2227^{* * *}$ & 0,0222 \\
0 & $0,0909^{* * *}$ & $0,0676^{* *}$ & 0,0001 \\
-1 & 0,4585 & 0,0034 & 0,1264 & 0,0000 \\
-2 & 0,0428 & 0,0000 & $0,1893^{* * *}$ & 0,1452 \\
-3 & $0,0836^{* * *}$ & 0,1015 & 0,0356 & 0,0000 \\
-5 & $0,0891^{* * *}$ & 0,0065 & $0,1998^{* * *}$ & 0,0000 \\
\end{tabular}

* Significativo a $10 \%$; ** Significativo a $5 \%$; ** Significativo a $1 \%$.

Fonte: Resultados da pesquisa.

Tabela 10. Teste de causalidade LM para as volatilidades à vista e futura dos contratos de café e boi entre janeiro de 2007 e dezembro de 2010

\begin{tabular}{ccccc}
\hline \multirow{2}{*}{ Hipóteses } & \multicolumn{2}{c}{ Café Arábica } & \multicolumn{2}{c}{ Boi Gordo } \\
\cline { 2 - 5 } & $\lambda_{\text {LM }}$ & $p$-valor & $\lambda_{\text {LM }}$ & $p$-valor \\
\hline Futuro não causa à vista & 4,4327 & 0,1090 & 7,0021 & $0,0302^{* *}$ \\
À vista não causa futuro & 4,9260 & $0,0853^{*}$ & 6,8326 & $0,0328^{* *}$ \\
\hline
\end{tabular}

* Significativo a $10 \%$; * Significativo a $5 \%$.

Fonte: Resultados da pesquisa.

tivas (futuro causa à vista) e nas defasagens negativas (à vista causa futuro). Os resultados foram semelhantes para o mercado de café, porém, a persistência da relação causal é menor. Os movimentos de um dia neste último mercado afetaram a variabilidade das cotações à vista do dia posterior, enquanto que o mercado à vista em $t-2 \mathrm{e}$ $t$ - 3 causou, em termos de variância, o mercado futuro em $t^{23}$.

A Tabela 10 indica os resultados do teste de Hafner e Herwartz (2006). A hipótese de que o mercado futuro influencia num aumento da variabilidade do mercado spot, e vice-versa, foi confirmada pelo teste LM no mercado de boi gordo. Verifica-se causalidade bidirecional nos

23. No mercado de café, foram também realizados testes com preços futuros de primeiro vencimento (ao invés de considerar somente os vencimentos setembro e dezembro). Os resultados foram similares. mercados spot e futuro, confirmando as análises da Seção 4.1. Porém, no mercado de café, a relação se mostrou unicausal do mercado spot para o mercado futuro, rejeitando a hipótese de que a volatilidade dos preços futuros impacta a variabilidade das cotações spot.

\section{Conclusões}

As negociações de contratos de derivativos sobre ativos financeiros e commodities nos mercados globais desencadearam um controverso debate na literatura econômica e financeira acerca da influência desses papéis sobre as cotações no mercado à vista, no sentido de alterar seu padrão de volatilidade, o que pode gerar oscilações capazes de elevar o risco do sistema financeiro em geral. Dessa forma, este trabalho avaliou se o 
aumento das negociações dos contratos futuros de commodities, especificamente de boi gordo e café arábica, transacionados na BM\&FBOVESPA, teve impacto na volatilidade das cotações à vista durante a década de 2000. Para isso, avaliou-se a causalidade entre o volume de negociações desses contratos e a volatilidade dos preços spot por meio do teste de causalidade de Granger e da decomposição da variância do erro de previsão, utilizando modelos de vetores autorregressivos. Foram também realizados testes de causalidade na variância entre os mercados futuro e à vista, baseados na análise da função de correlação cruzada, proposto por Cheung e Ng (1996), e na consideração do conceito de multiplicadorde Lagrange, desenvolvido por Hafner e Herwartz (2006).

Os resultados do teste de causalidade de Granger indicaram bicausalidade entre a volatilidade dos preços à vista e o componente não esperado do volume de contratos negociados nos mercados de café arábica e de boi gordo. Considerando-se a decomposição da variância do erro de previsão, em um período de 21 dias úteis, observou-se que a volatilidade spot teve influência do componente não esperado do volume negociado, especialmente no mercado de café. Verificou-se, ainda, com destaque para o mercado de boi gordo, elevada influência da volatilidade dos preços futuros sobre a volatilidade spot. Este último fato foi, inclusive, captado pelos testes de causalidade na variância.

Dessa forma, pode-se inferir que, nos anos 2000, a negociação de contratos futuros se constituiu em mais um fator explicativo para o entendimento do comportamento da volatilidade das cotações à vista do café arábica e do boi gordo no Brasil, além das questões conjunturais associadas à perda do poder de compra da moeda norte-americana e, especialmente, dos aspectos estruturais relativos à oferta e demanda de tais produtos. Sobre a última questão, convém ressaltar que os padrões de consumo e de produção nos mercados locais e globais de commodities agropecuárias, incluindo as analisadas neste estudo, registraram alterações; alterações estas que expli- cam, em grande medida, as oscilações dos preços à vista e futuros. Além disso, vale observar que tais aspectos estruturais também acabam por influenciar na decisão de uso ou não de derivativos para gestão de risco ou para especulação, que, por consequência, interferem na evolução dos negócios com estes papéis.

Além da relevância em termos acadêmicos, os resultados podem ser úteis aos participantes dos mercados de boi gordo e café arábica na medida em que as relações de causalidade evidenciadas podem auxiliar nas estratégias de gerenciamento de riscos, bem como na elaboração de modelos de descoberta de preços. Extensões dessas análises podem incluir a avaliação da causalidade por meio de métodos multivariados para estimação das volatilidades, assim como considerar técnicas baseadas em funções de densidade de probabilidade para testes de hipótese de alterações de padrões.

\section{Referências bibliográficas}

ADRANGI, B. e CHATRATH, A. Futures commitments and exchange rate volatility. Journal of Business Finance and Accounting, v. 25, n. 3, p. 501-520, 1998.

AHMAD, H., SHAH, S. Z. A. e SHAH, I. A. Impact of Futures Trading on Spot Price Volatility: Evidence from Pakistan. International Research Journal of Finance and Economics, v. 59, p. 145-165, 2010.

ALEXAKIS, P. On the effect of index futures trading on stock market volatility. International Research Journal of Finance and Economics, v. 11, p. 7-20, 2007.

ANTONIOU, A. e FOSTER, A. J. The effect of futures trading on spot price volatility: Evidence for Brent crude oil using GARCH. Journal of Business Finance and Accounting, v. 19, n. 4, p. 473-484, 1992.

ANTONIOU, A. e HOLMES, P. Futures trading, information and spot price volatility: Evidence for the FTSE-100 Stock Index Futures contract using GARCH. Journal of Banking \& Finance, v. 19, n. 1, p. 117-129, 1995.

ANTONIOU, A., HOLMES, P. e PRIESTLEY, R. The effects on stock index futures trading on stock index volatility: An analysis of the asymmetric response of volatility to news. The Journal of Futures Market, v. 18, n. 2, p. 151-166, 1998. 
BAE, S. C., KWON, T. H. e PARK, J. W. Derivatives trading, volatility spillover, and regulation: Evidence from the Korean securities markets. The Journal of Futures Markets, v. 29, n. 6, p. 563-597, 2009.

BANDIVADEKAR, S. e GHOSH, S. Derivatives and volatility on Indian stock markets. Reserve Bank of India Occasional Papers, v. 24, n. 3, p. 1-15, 2003.

BESSEMBINDER, H. e SEGUIN, P. Futures trading activity and stock price volatility. Journal of Finance, v. 47, n. 5, p. 2015-34, 1992.

BESSEMBINDER, H., CHAN, H. e SEGUIN, P. An empirical examination of information, differences of opinion, and trading activity. Journal of Financial Economics, v. 40, n. 1, p. 105-134, 1996.

BOARD, J., SANDMANN, G. e SUTCLIFFE, C. The effect of futures market volume on spot market volatility. Journal of Business Finance \& Accounting, v. 28, n. 7 e 8, p. 799-819, 2001.

BOLLERSLEV, T. Generalized autoregressive conditional heteroscedasticity. Journal of Econometrics, v. 31, n. 3, p. 307-327, 1986.

BOLOGNA, P. e CAVALLO, L. Does the introduction of stock index futures effectively reduce stock market volatility? Is the 'futures effect' immediate? Evidence from the Italian stock exchange using GARCH, Applied Financial Economics, v. 12, n. 3, p. 183-92, 2002.

BRORSEN, B. W., OELLERMANN, C. M. e FARRIS. P. L. The live cattle futures market and daily cash price movements. The Journal of Futures Markets, v. 9, n. 4, p. 273-282, 1989.

BROWN, C. J. e CURCI, R. Mexican peso futures and exchange rate volatility. Latin American Business Review, v. 3, n. 1, p. 75-90, 2002.

CHATRATH, A., RAMCHANDER, S. e SONG, F. The role of futures trading activity in exchange rate volatility. The Journal of Futures Markets, v. 16, n. 5, p. 561-584, 1996.

CHEUNG, Y. W. e NG, L. K. A causality in variance test and its application to financial market prices. Journal of Econometrics, v. 72, p. 33-48, 1996.

CLIFTON, E. V. The currency futures market and interbank foreign exchange trading. The Journal of Futures Markets, v. 5, n. 3, p. 375-384, 1985.

COX, C. C. Futures trading and market information. Journal of Political Economy, v. 84, n. 6, p. 1215-1237, 1976.

DAWSON, P. e STAIKOURAS, S. K. The impact of volatility derivatives on S\&P 500 volatility. The Journal of Futures Markets, v. 29, n. 12, p. 1190-1213, 2009.
DEBASISH, S. S. Effect of futures trading on spot-price volatility: Evidence for NSE Nifty using GARCH. The Journal of Risk Finance, v. 10, n. 1, p. 67-77, 2009.

DRIMBETAS, E., SARIANNIDIS, N. e PORFIRIS, N. The effect of derivatives trading on volatility of the underlying asset: Evidence from the Greek stock market. Applied Financial Economics, v. 17, n. 2, p. 139148, 2007.

EMERY, H. C. Speculation on the stock and produce exchanges of the United States. Columbia University, New York, 1896.

ENGLE, R. F. Autoregressive conditional heteroscedasticity with estimates of the variance of United Kingdom inflation. Econometrica, v. 50, n. 4, p. 987-1007, 1982.

FIGLEWSKI, S. Futures trading and volatility in the GNMA market. Journal of Finance, v. 36, p. 445-456, 1981.

GALVÃO, A. B., PORTUGAL, M. S. e RIBEIRO, E. P. Volatilidade e causalidade: evidências para o mercado à vista e futuro de índice de ações no Brasil. Revista Brasileira de Economia, v. 54, n. 1, p. 37-56, 2000.

GARCIA, P., LEUTHOLD, R. M. e ZAPATA, H. Leadlag relationships between trading volume and price variability: new evidence. The Journal of Futures Markets, v. 6, n. 1, p. 1-10, 1986.

GRAY, R. W. Onions revisited. Journal of Farm Economics, v. 65, n. 2, p. 273-276, 1963.

HAFNER, C. M. e HERWARTZ, H. A Lagrange multiplier test for causality in variance. Economics Letters, v. 93, p. 137-141, 2006.

HEGDE, S. P. The impact of futures trading on the spot market for treasury bonds. Financial Review, v. 29, n. 4, p. 441-471, 1994.

HOOKER, R. H. The suspension of the Berlin produce exchange and its effect upon corn prices. Journal of the Royal Statistical Society, v. 64, n. 4, p. 574-604, 1901.

ILLUECA, M. e LAFUENTE, J. A. The effect of spot and futures trading on stock index market volatility: A nonparametric approach. The Journal of Futures Markets, v. 23, n. 9, p. 841-858, 2003.

ILLUECA, M. e LAFUENTE, J. A. Introducing the mini-futures contract on Ibex 35: implications for price discovery and volatility transmission. Spanish Economic Review, v. 10, n. 3, p. 197-219, 2008.

JENSEN, G. R., JOHNSON, R. R. e MERCER, J. M. Tactical asset allocation and commodity futures. Journal of Portfolio Management, v. 28, n. 4, p. 100-111, 2002. 
432 Derivativos sobre Commodities Influenciam a Volatilidade dos Preços à Vista? Uma análise nos mercados de boi gordo e café arábica no Brasil

JOCHUM, C. e KODRES, L. Does the introduction of futures on emerging market currencies destabilize the underlying currencies? IMF Staff Papers, v. 45, n. 3, p. 486-521, 1998.

JOHNSON, A. C. Effects of futures trading on price performance in the cash onion market, 1930-68, Washington: U.S. Department of Agriculture, Technical Bulletin no 1470, 1973

KASMAN, A. e KASMAN, S. The impact of futures trading on volatility of the underlying asset in the Turkish stock market. Physica A: Statistical Mechanics and its Applications, v. 387, n. 12, p. 2837-2845, 2008.

KOCAGIL, A. E. Does futures speculation stabilize spot prices? Evidence from metals markets. Applied Financial Economics, v. 7, p. 115-125, 1997.

KOCAGIL, A. E. e SHACHMUROVE, Y. Return Volume dynamics in futures markets. The Journal of Futures Markets, v. 18, n. 4, p. 399-426, 1998.

MALLIARIS, A. G. e URRUTIA, J. L. Volume and price relationships: hypotheses and testing for agricultural futures. The Journal of Futures Markets, v. 18, n. 1, p. 53-72, 1998.

MAYHEW, S. The impact of derivatives on cash markets: what have we learned? Working paper, Department of Banking and Finance, Terry College of Business, University of Georgia, 2000.

MORGAN, C. W. Futures markets and spot price volatility: A case study. Journal of Agricultural Economics, v. 50, n. 2, p. 247-257, 1999.

MORIARTY, E. J. e TOSINI, P. A. Futures trading and the price volatility of GNMA certificates-further evidence. The Journal of Futures Markets, v. 5, n. 4, p. 633-641, 1985.

NETZ, J. S. The effect of futures markets and corners on storage and spot price variability. American Journal of Agricultural Economics, v. 77, n. 1, p. 182-193, 1995.

PECK, A. E. Reflections of hedging on futures market activity. Food Research Institute Studies, v. 17, n. 3, p. 327349, 1979.

PHILLIPS, P. C. B. e PERRON, P. Testing for a unit root in time series regression. Biometrika, v. 75, p. 335-346, 1988.

PILAR, C. e RAFAEL, S. Does derivatives trading destabilize the underlying assets? Evidence from the Spanish stock market. Applied Economics Letters, v. 9, n. 2, p. 107-110, 2002.

POK, W. C. e POSHAKWALE, S. The impact of futures contracts on the spot market volatility: The case of
Kuala Lumpur Stock Exchange. Applied Financial Economics, v. 14, n. 2, p. 143-154, 2004.

POWERS, M. J. Does futures trading reduce price fluctuations in the cash markets? American Economic Review, v. 60, n. 3, p. 460-464, 1970.

RAO, R. Impact of financial derivative products on spot market volatility: A study of Nifty. The ICFAI Journal of Derivatives Market, v. 4, n. 1, p. 7-16, 2007.

REDRADO, M., CARRERA, J., BASTOURRE, D. e IBARLUCÍA, J. Financialization of commodity markets: Nonlinear consequences from heterogeneous agent behavior. Working Paper 44, Banco Central de la República Argentina, 2009.

RYOO, H-J. e SMITH, G. The impact of stock index futures on the Korean stock market. Applied Financial Economics, v. 14, n. 4, p. 243-251, 2004.

SANTOS, J. Did futures markets stabilize US grain price? Journal of Agricultural Economics, v. 53, n. 1, p. 25-36, 2002.

SHASTRI, K., SULTAN, J. e TANDON, K. The impact of the listing of options in the foreign exchange market. Journal of International Money and Finance, v. 15, n. 1, p. 37-64, 1996.

SPYROU, S. I. Index futures trading and spot price volatility. Journal of Emerging Market Finance, v. 4, n. 2, p. 151-167, 2005.

SRINIVASAN, P. e BHAT, K. S. The impact of futures trading on the spot market volatility of selected commercial banks in India. European Journal of Economics, Finance and Administrative Sciences, n. 14, p. 28-40, 2008.

STAIKOURAS, S. K. Testing the stabilization hypothesis in the UK short-term interest rates: evidence from a GARCH-X model. Quarterly Review of Economics and Finance, v. 46, n. 2, p. 169-189, 2006.

STEIN, J. Informational externalities and welfarereducing speculation. Journal of Political Economy, v. 95, p. $1123-1145,1987$.

TAYLOR, G. S. e LEUTHOLD, R. M. The influence of futures trading on cash cattle price variations. Food Research Institute Studies, v. 13, n. 1, p. 29-35, 1974.

THRAEN, C. S. The emerging futures market for cheddar cheese: A mechanism for stability or increased spot-price volatility? Proceedings of the NCR-134 Conference on Applied Commodity Price Analysis, Forecasting, and Market Risk Management, Chicago, IL, 1998. 
TOMEK, W. G. A note on historical wheat prices and futures trading. Food Research Institute Studies, v. 110, n. 1, p. 109-113, 1971.

TRIPATHY, N., RAO, S. V. R. e KANAGARAJ, A. Impact of derivatives trading on spot market volatility: An empirical study. International Journal of Applied Decision Sciences, v. 2, n. 2, p. 209-232, 2009.

UNCTAD. The financialization of commodity markets. In: Trade and Development Report, United Nations, New York and Geneva, 2009.

URSO, F. S. P. A cadeia da carne bovina no Brasil: Uma análise de poder de mercado e teoria da informação. Tese apresentada à Escola de Economia de São Paulo, Fundação Getúlio Vargas, FGV-SP, 2007.

WEAVER, R. D. e BANERJEE, A. Does futures trading destabilize cash prices? Evidence for $\mathrm{U}, \mathrm{S}$, live beef cattle. The Journal of Futures Markets, v. 10, n. 1, p. 41-60, 1990.

WORKING, H. Price effects of futures trading. Food Research Institute Studies, v. 1, n. 1, p. 3-31, 1960.

WRAY, L. R. O novo capitalismo dos gerentes de dinheiro e a crise financeira global. Oikos, v. 8, n. 1, p. 19-39, 2009.

YANG, J., BALYEAT, R. B. e LEATHAM, D. J. Futures trading activity and commodity cash price volatility. Journal of Business Finance \& Accounting, v. 32, n. 1 e 2, p. 297-323, 2005.

ZHONG, M., DARRAT, A. F. e OTERO, R. Price discovery and volatility spillovers in index futures markets: Some evidence from Mexico. Journal of Banking \& Finance, v. 28, n, 12, p. 3037-3054, 2004. 
434 - Derivativos sobre Commodities Influenciam a Volatilidade dos Preços à Vista? Uma análise nos mercados de boi gordo e café arábica no Brasil

\section{Anexos}

Anexo 1. Evolução dos preços (à vista e futuro) e dos contratos (negociados e em aberto) no mercado de café entre janeiro de 2000 e dezembro de 2010

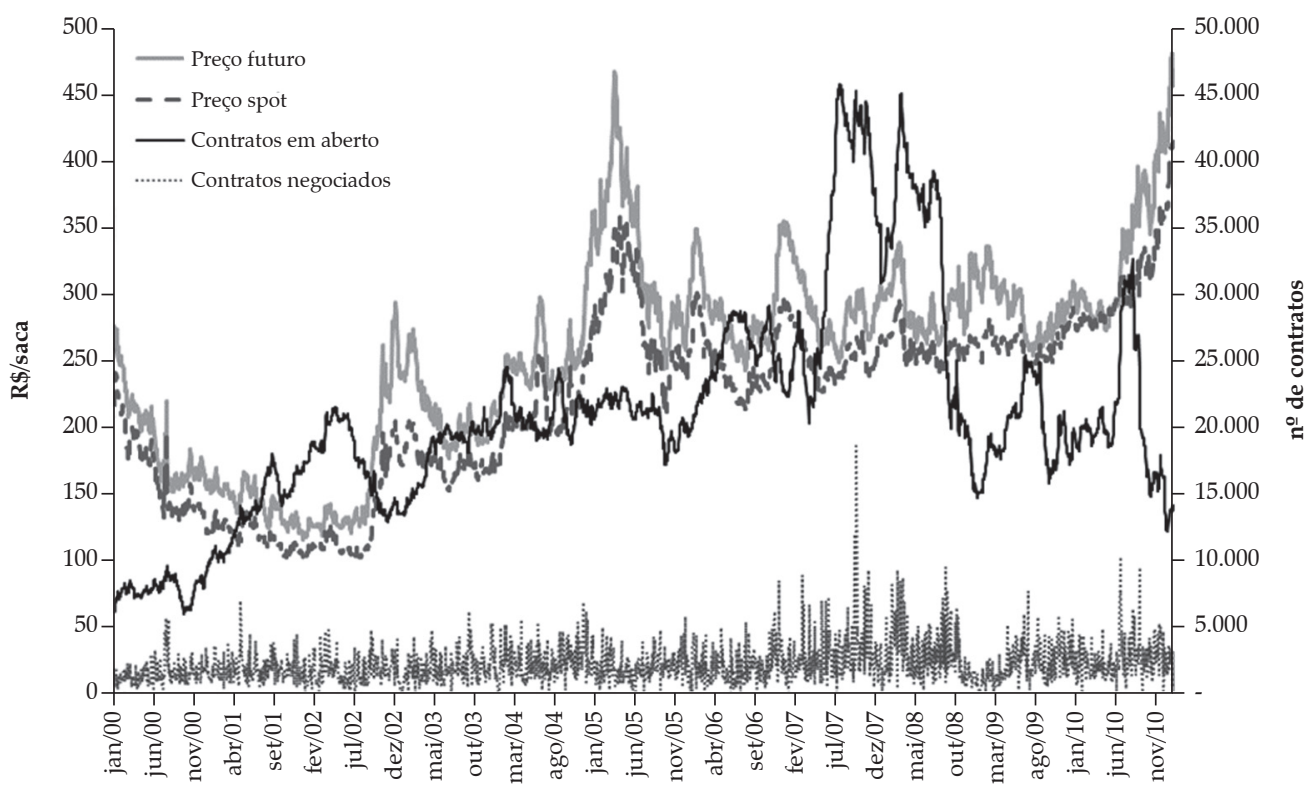

Fonte: BM\&FBOVESPA.

Anexo 2. Evolução dos preços (à vista e futuro) e dos contratos (negociados e em aberto) no mercado de boi gordo entre janeiro de 2001 e dezembro de 2010

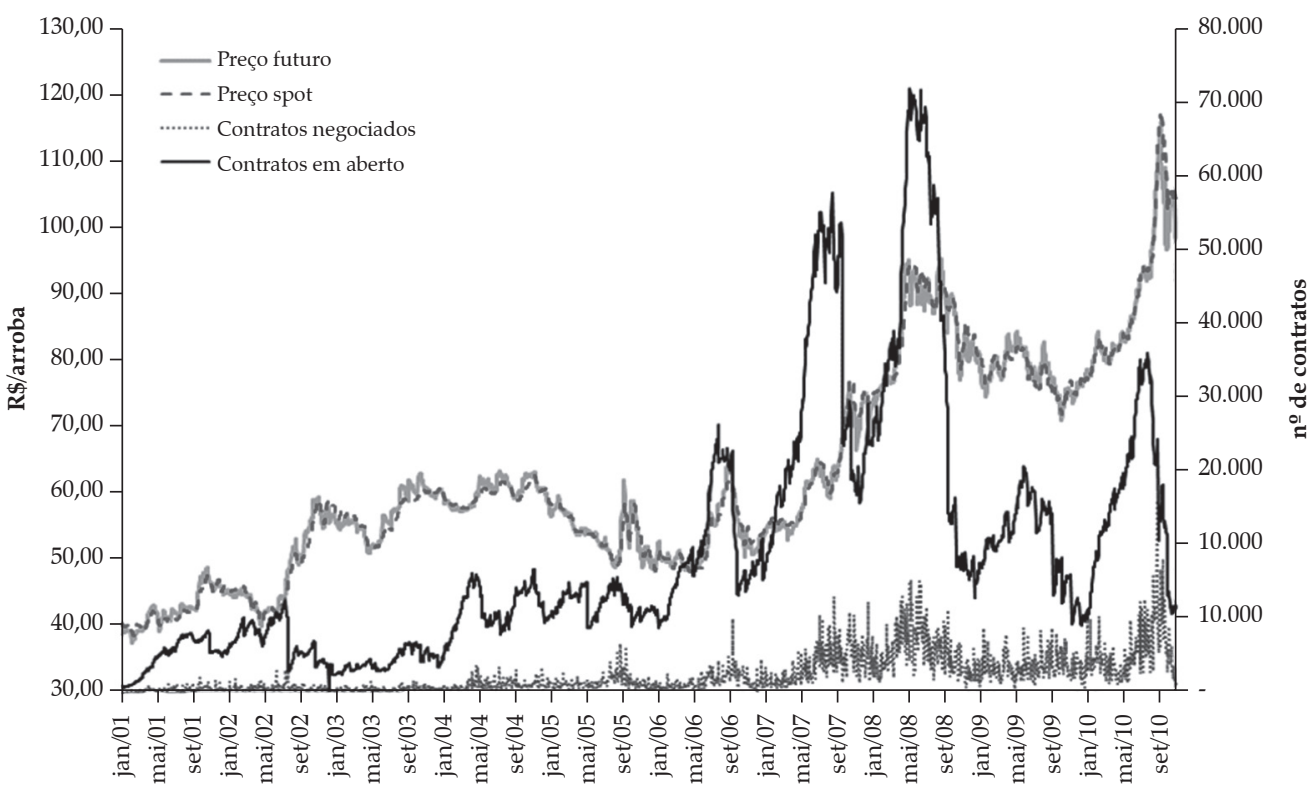

Fonte: BM\&FBOVESPA. 
Anexo 3. Evolução da volatilidade no mercado à vista e do componente não esperado dos contratos (negociados e em aberto) no mercado de café entre janeiro de 2000 e dezembro de 2010

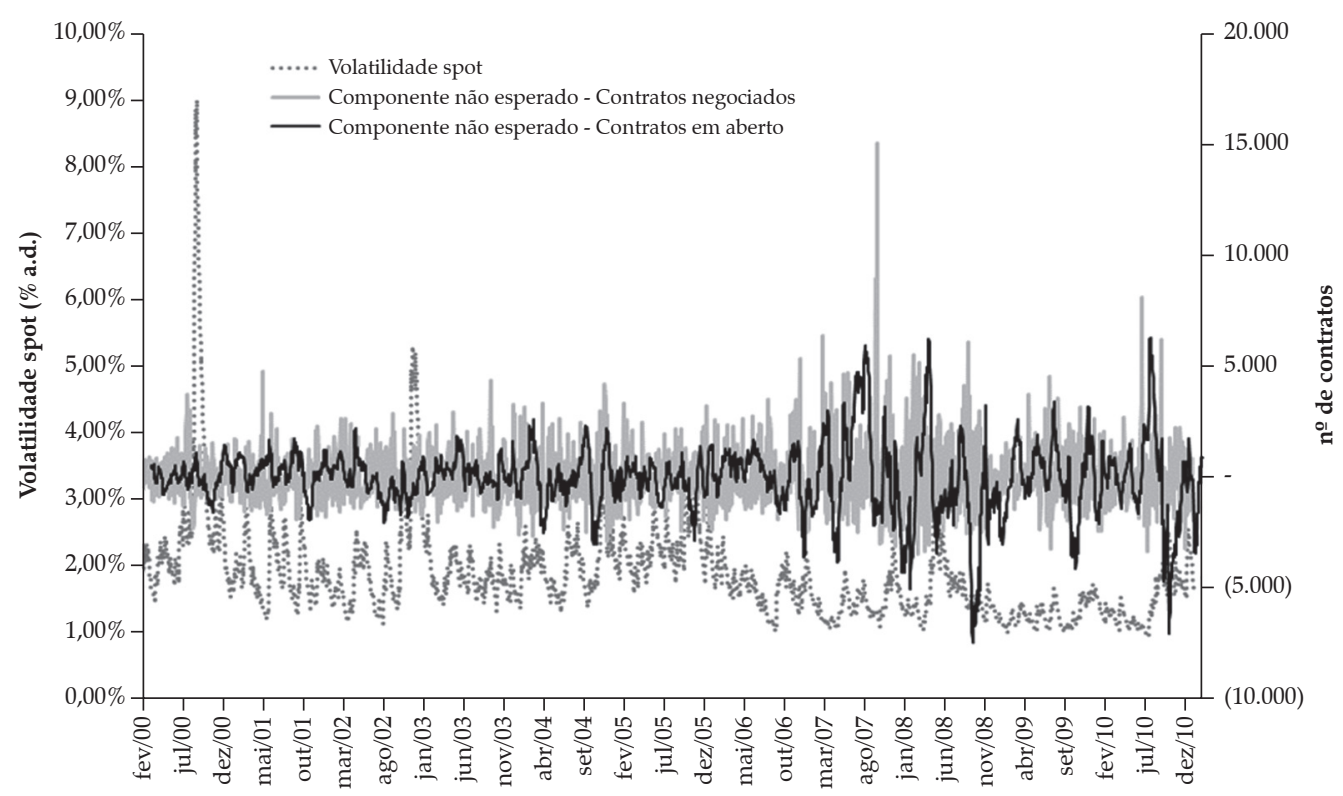

Fonte: Resultados da pesquisa.

Anexo 4. Evolução da volatilidade no mercado à vista e do componente não esperado dos contratos (negociados e em aberto) no mercado de boi gordo entre janeiro de 2001 e dezembro de 2010

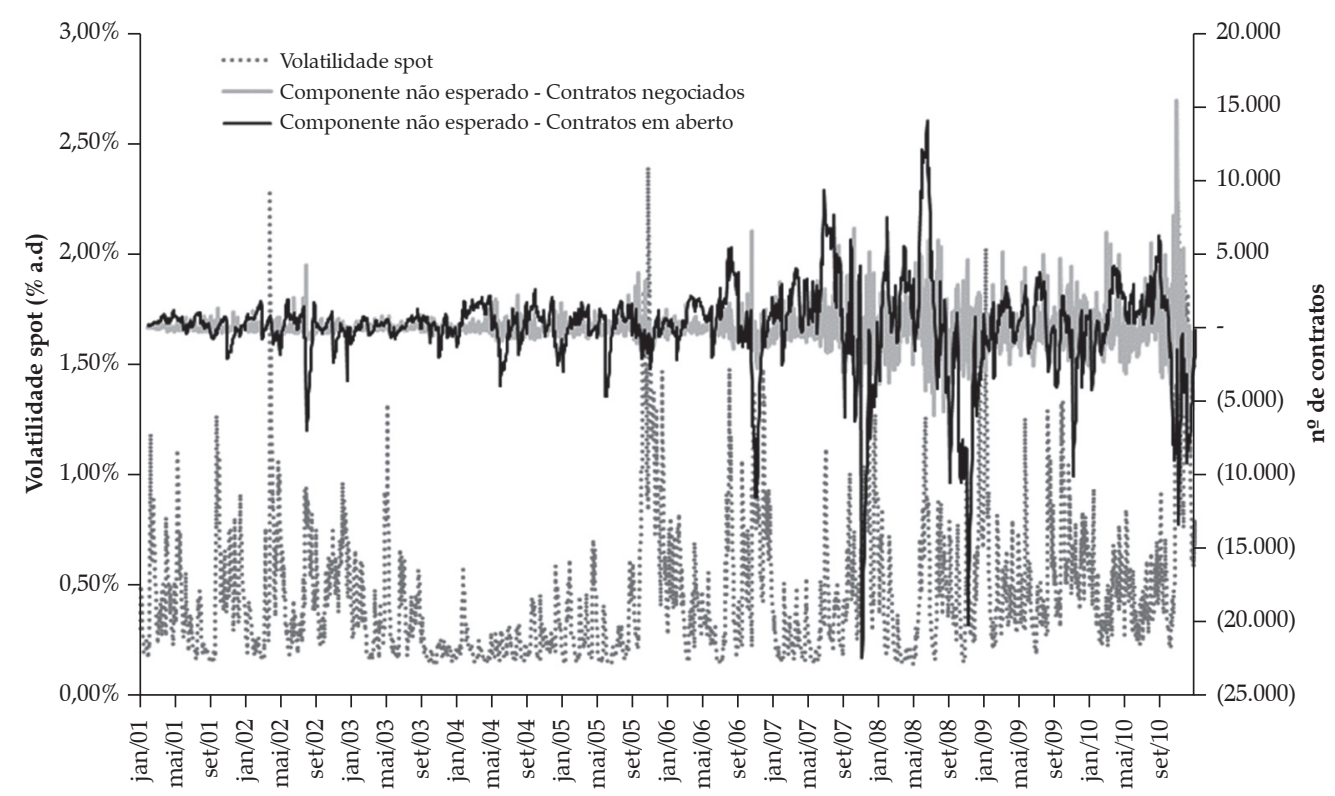

Fonte: Resultados da pesquisa. 
436 - Derivativos sobre Commodities Influenciam a Volatilidade dos Preços à Vista? Uma análise nos mercados de boi gordo e café arábica no Brasil

Anexo 5. Testes de raízes unitárias para as séries de café - procedimento de Phillips-Perron*

\begin{tabular}{|c|c|c|c|c|c|c|c|}
\hline \multirow[t]{2}{*}{ Mercados } & \multirow[t]{2}{*}{ Modelo } & $\begin{array}{c}\text { Estatística } \\
\text { estimada }\end{array}$ & \multirow[t]{2}{*}{$p$-valor } & $\begin{array}{c}\text { Estatística } \\
\text { estimada }\end{array}$ & \multirow[t]{2}{*}{$p$-valor } & $\begin{array}{c}\text { Estatística } \\
\text { estimada }\end{array}$ & \multirow[t]{2}{*}{$p$-valor } \\
\hline & & CNECN & & CNECA & & VolSpot & \\
\hline \multirow{3}{*}{$\begin{array}{l}\text { Café } \\
\text { Arábica }\end{array}$} & Com constante e tendência linear & $-4,09$ & 0,00 & $-8,35$ & 0,00 & $-7,24$ & 0,00 \\
\hline & Com constante & $-4,09$ & 0,00 & $-8,32$ & 0,00 & $-6,45$ & 0,00 \\
\hline & Sem termos deterministas & $-4,10$ & 0,00 & $-8,32$ & 0,00 & $-2,34$ & 0,02 \\
\hline \multirow{3}{*}{$\begin{array}{l}\text { Boi } \\
\text { Gordo }\end{array}$} & Com constante e tendência linear & $-39,31$ & 0,00 & $-8,57$ & 0,00 & $-13,71$ & 0,00 \\
\hline & Com constante & $-39,31$ & 0,00 & $-8,55$ & 0,00 & $-13,45$ & 0,00 \\
\hline & Sem termos deterministas & $-39,32$ & 0,00 & $-8,55$ & 0,00 & $-11,42$ & 0,00 \\
\hline
\end{tabular}

* Hipótese nula: existência de uma raiz unitária.

Fonte: Resultados da pesquisa.

Anexo 6. Seleção do VAR - número de defasagens

\begin{tabular}{|c|c|c|c|c|c|c|c|}
\hline \multirow{2}{*}{ Mercado } & \multirow{2}{*}{ Lag } & \multicolumn{3}{|c|}{ Modelo VAR com VolSpot e CNECN } & \multicolumn{3}{|c|}{ Modelo VAR com VolSpot e CNECA } \\
\hline & & AIC & SC & $H Q$ & AIC & SC & $H Q$ \\
\hline \multirow{11}{*}{$\begin{array}{l}\text { Café } \\
\text { Arábica }\end{array}$} & 0 & 10,1183 & 10,1227 & 10,1199 & 10,5458 & 10,5502 & 10,5474 \\
\hline & 1 & 6,7385 & 6,7516 & 6,7432 & 4,6149 & 4,6280 & 4,6196 \\
\hline & 2 & 6,7218 & 6,7437 & 6,7297 & 4,5883 & 4,6102 & 4,5962 \\
\hline & 3 & 6,7191 & 6,7498 & 6,7302 & 4,5766 & 4,6073 & 4,5877 \\
\hline & 4 & 6,7158 & 6,7553 & 6,7301 & 4,5743 & 4,6137 & 4,5885 \\
\hline & 5 & 6,6906 & $6,7388^{*}$ & $6,7080^{*}$ & 4,5585 & $4,6067^{*}$ & $4,5759^{*}$ \\
\hline & 6 & 6,6906 & 6,7476 & 6,7112 & 4,5577 & 4,6147 & 4,5783 \\
\hline & 7 & 6,6921 & 6,7579 & 6,7159 & 4,5596 & 4,6254 & 4,5834 \\
\hline & 8 & 6,6923 & 6,7668 & 6,7192 & 4,5615 & 4,6360 & 4,5885 \\
\hline & 9 & $6,6897^{*}$ & 6,7730 & 6,7199 & $4,5575^{*}$ & 4,6408 & 4,5876 \\
\hline & 10 & 6,6916 & 6,7837 & 6,7249 & 4,5595 & 4,6515 & 4,5928 \\
\hline \multirow{11}{*}{$\begin{array}{l}\text { Boi } \\
\text { Gordo }\end{array}$} & 0 & 1,7997 & 1,8471 & 0,1817 & 1,8311 & 1,8359 & 1,8329 \\
\hline & 1 & $-1,2879$ & $-1,2737$ & $-1,2828$ & $-1,7385$ & $-1,7243^{*}$ & $-1,7333^{*}$ \\
\hline & 2 & $-1,2963$ & $-1,2725$ & $-1,2876$ & $-1,7392^{*}$ & $-1,7155$ & $-1,7306$ \\
\hline & 3 & $-1,3070$ & $-1,2737$ & $-1,2949$ & $-1,7372$ & $-1,7040$ & $-1,7251$ \\
\hline & 4 & $-1,3141$ & $-1,2714$ & $-1,2986$ & $-1,7355$ & $-1,6928$ & $-1,7200$ \\
\hline & 5 & $-1,3369$ & $-1,2846^{*}$ & $-1,3179$ & $-1,7352$ & $-1,6829$ & $-1,7162$ \\
\hline & 6 & $-1,3460$ & $-1,2843$ & $-1,3236^{*}$ & $-1,7378$ & $-1,6761$ & $-1,7154$ \\
\hline & 7 & $-1,3458$ & $-1,2746$ & $-1,3199$ & $-1,7370$ & $-1,6658$ & $-1,7111$ \\
\hline & 8 & $-1,3444$ & $-1,2637$ & $-1,3151$ & $-1,7346$ & $-1,6539$ & $-1,7053$ \\
\hline & 9 & $-1,3490^{*}$ & $-1,2588$ & $-1,3162$ & $-1,7386$ & $-1,6484$ & $-1,7058$ \\
\hline & 10 & $-1,3483$ & $-1,2486$ & $-1,3121$ & $-1,7382$ & $-1,6385$ & $-1,7020$ \\
\hline
\end{tabular}

* Indica menor valor do critério de informação.

Fonte: Resultados da pesquisa. 